\title{
A Semi-blind Model with Parameter Identification for Building Temperature Estimation
}

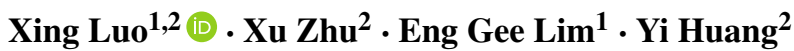

Received: 21 February 2017 / Accepted: 31 May 2017 / Published online: 29 June 2017

(C) The Author(s) 2017. This article is an open access publication

\begin{abstract}
An accurate thermal model for building enables the heating system (HS) to work efficiently as well as save energy. Thermal modelling often requires physical parameters of the building, which are difficult to be accurately determined. The aim of this work is to develop an optimal thermal model for better understanding of thermal dynamics with the goal of using this to estimate temperature variation in a few hours ahead within building. Based on the characteristics of thermal motion, a conventional physics-based (PB) model for building temperature estimation is introduced first. Afterwards, in order to refine the model and improve the actual performance, we propose an innovative semi-blind (SB) model based on data-driven approaches. Additionally, the methodologies including self-adaptive algorithms (SAAs) and grey prediction technique (GPT) have been applied in dealing with the integrated parameters estimation (IPE) process to ensure the practicability of the implemented model. The proposed model schema is validated by testing in a laboratory. The results indicate that the proposed approach achieves much higher accuracy in estimating temperature variation than the conventional PB model, with only limited knowledge of the building characteristics. The root mean square deviation (RMSD) of SB model and PB model are 0.18 and 0.43 , respectively. According to the results, it can be conclu-
\end{abstract}

Xing Luo

xing.luo@xjtlu.edu.cn; sgxluo2@liverpool.ac.uk

$\bowtie \mathrm{XuZhu}$

xuzhu@liverpool.ac.uk

1 Department of Electrical and Electronic Engineering, Xi'an Jiaotong-Liverpool University, Suzhou, 215123, China

2 Department of Electrical Engineering and Electronics, University of Liverpool, Liverpool, L69 3GJ, UK ded that the proposed SB model is able to appropriately estimate the internal temperature values and great improvement has been achieved comparing with the original thermal model.

Keywords Thermal modelling · Physics-based (PB) model $\cdot$ Semi-blind (SB) model $\cdot$ Parameter estimation

\section{Introduction}

Energy shortage is becoming one of the most significant worldwide open issues in the past decades [1]. The most effective and least controversial way to resolve this problem is to rationally allocate energy resources and thus to reduce waste of resources [2]. In particular, among the total energy usage in domestic buildings, the heating system (HS), which is one of the most common used devices, plays a significant role not only in keeping house maintaining warm but also in energy consumption. According to statistics, it accounts for over $40 \%$ of the total energy consumption which is an impressive proportion during winter time in a UK family ("[online] available: http://www.britishgas.co.uk/") [3]. For this reason, it is necessary to make people aware of their house energy consumption in advance; thus, actions can be taken to reduce utility bill. Figure 1 depicts the operational principle of a conventional HS. The programmer embedded in boiler can set the start-end time for the hot water and heating in the house over a day. The thermostat is able to monitor the real-time local temperature and turns the heating off automatically once the pre-set target temperature is achieved.

However, without loss of generality, we take a typical UK domestic heating system as an example of investigation. Until now, the often operation is only to set the timer according to the habits of the residents without taking the 


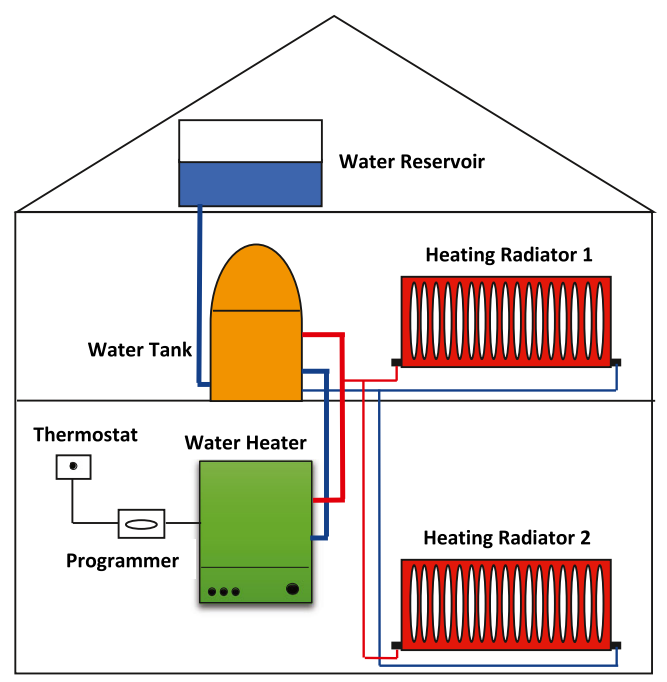

Fig. 1 A conventional house heating control system. The boiler supplies hot water for daily use and heating

surrounding environment conditions into account-it does not matter whether the external temperature is high or low. Nonetheless, if outside is chilly cold, the room will be still cold at the required time. On the contrary, the room temperature will be ready well before the desired time if outside is warm enough. Therefore, these invariable settings of HS will result in considerable energy waste and negative using experiences. In order to deal with this issue, one effective approach is to build a thermal model of the building, that can be used to reflect the thermal dynamics property of the building $[4,5]$. Thereupon, the regularity of the temperature variation of the building can be obtained, and the operating time of HS can be scheduled properly and reasonably. This should ensure the right temperature for the right periods of time and reduce the waste of energy in a way.

Much researches have been conducted on thermal model of building in recent years, and there are many popular house thermal models being presented in literatures. In [6], in order to describe the thermal dynamics of building, the authors proposed an evolutionary approach to parameter identification for thermal models. In this way, the parameter estimation is formulated as several optimisation tasks. In [7], the authors introduced a 1R1C lumped parameter circuit which presents a building thermal model using thermal electric analogy on the basis of the similarities between the RC circuit and the house thermal dynamics. Nonetheless, the related parameters are difficult to be determined due to the measurement. In addition, a proposed least-absolute shrinkage and selection operator (LASSO)-based model for a heating, ventilation and air-conditioning (HVAC) system can be found in [8]. The proposed approach leads to a linear thermal model as against the non-linear building models and makes it easier to design model-based controller. Furthermore, in [3], an autoregressive model with exogenous inputs (ARX model) was used to identify the house thermal model, and it can be used to predict the energy consumption of HVAC system at home.

Additionally, self-adaptive algorithms have wide applications in many research fields and some popular selfadaptive algorithms can be found in literatures. In [9], the authors proposed a self-adaptive extreme learning machine (SELM) algorithm to find the proper and universal number of hidden nodes in single hidden layer feedforward networks (SLFNs). Moreover, a self-adaptive window that is autonomously adjusted through a statistical hypothesis test was presented for handling complex concept drift in [10]. Furthermore, an adaptive dynamic programmingbased algorithm is introduced in [11], to manage load demand and minimise the entire energy cost using renewable energy in smart grid. The previous work is significant and can be used for reference in establishing an optimal thermal model in our work.

In this paper, we propose two thermal models referred to as physics-based (PB) model and semi-blind (SB) model, for temperature estimation within building in hours ahead. Additionally, SB model is developed based on PB model. Compared with the previous researches, the contribution of this work can be summarised as in following aspects. (i) In this paper, the cognitive computing methods are used in a new field that to establish a house thermal model and by using this to estimate the temperature variations within the building. (ii) In contrast to traditional cognitive computing methods which require a large amount of data for training, the proposed SB model is able to simulate the thermal dynamics and accurately estimate the temperature only based on first several days' data. (iii) Since the thermal dynamics within a building is affected by various physical parameters and some of them are difficult to be precisely determined in practice, we propose a highly integrated parameter identification method in conjunction with self-adaptive algorithms (SAAs) and grey prediction technique (GPT) for parameter estimation. Hence, the model can be continuously renewed along with experiencing time increasing. (iv) The experimental results indicate that great improvement has been achieved. The proposed SB model can appropriately estimate the temperature values with lower RMSD of 0.18 , which is better than 0.42 achieved by the conventional PB model.

The work in this paper is significant and worthy of researching since very few work in literatures use datadriven approaches in solving a problem like establishing a thermal model within building. Additionally, an optimum thermal model is the basis of better scheduling HS, and it can be used to reduce the energy waste and improve users experiences simultaneously, which connect us all.

The rest of paper is organised as follows: Section "Overview of the Building Thermal Model" presents a brief 
overview of the adopted thermal models. In "Physics-Based (PB) Estimation Model", a conventional PB model will be introduced first. Afterwards, in order to improve the estimation performance, the SB model and the relevant parameter estimation methods are discussed in "Semi-blind (SB) Estimation Model". Moveover, in "Experimental Study", a number of simulations are carried out to evaluate the feasibilities of the proposed models. The simulation results are compared with measured temperature data. The results demonstrate that the proposed SB model has great accuracy in evaluation of thermal dynamics features within buildings and can complete the task of temperature estimating. Finally, we conclude the paper concisely in "Conclusion".

\section{Overview of the Building Thermal Model}

When developing strategies to minimise energy consumption from HS within buildings, it is crucial to understand the dynamics of energy generation and loss [12-14]. However, the energy generation and loss have intimate connections with a number of most important factors, such as power rate of heat source, building characteristics and surrounding environment conditions $[15,16]$. Therefore, the proposed thermal model in this study is aimed to investigate the inner relationships between energy generation and loss, and the thermal response in temperature variation according to the provided information. The developed model will have adjustable parameters corresponding to different contributions to the heat budget and so by accurately understanding the form of the thermal dynamics in the building. In addition, an individual compartment or a number of neighbouring compartments with similar climate conditions are regarded as the smallest unit of the building in this study.

To investigate the impacts of the exterior factors, it is crucial to understand the most significant causes of heat generation and loss during thermal transferring process within a building. The block diagram in Fig. 2 gives an overview of an independent thermal model. The established thermal model receives information from exterior in order to estimate the internal temperature variations and compares with the actual temperature within a building. Based on Fig. 2, to develop such a model to be more closely aligned with the realistic, the information in following aspects should be considered:

- Building characteristics: spacial structures, volumes, areas, construction materials etc.;

- Internal/external environment information: temperature, wind speed, humidity, solar radiation, etc.;

- Heat source properties: heater type, power rate, efficiency and heat generated by other stuffs.

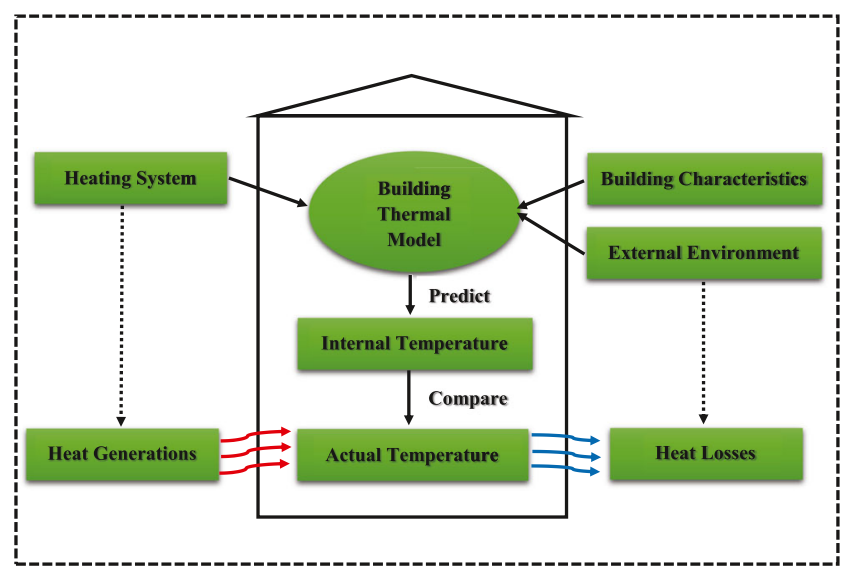

Fig. 2 The overview of an independent thermal model

In this independent thermal balance system, the heat sources consist of building's HS which is regarded as the main heat producer, and other factors that are from lighting, human activities etc. However, compared with the heat provided by the HS, the other effects may have limited impacts on internal temperature variation. Thus, they are treated as the disruptive factors and are restricted artificially in the testing experiments to ensure the accuracy of the thermal model evaluation. The HS is taken as the core heat source in this study.

In contrast to the heat generation, the heat loss happens in every second. For the heat loss, it can be sorted into two main forms in general: ventilation and conduction. On the one hand, the ventilation represents the air exchange through cracks and gaps between joints. However, the finest airtightness of the building is assumed in this study so that the heat loss which is caused by ventilation can be neglected. On the other hand, the conductive heat loss can be thought in terms of heating flowing from a hot region to a cold region through walls, windows and other conductive components. Since the heat conduction happens at all times when there are temperature differences between interior and exterior, it makes a significant contribution in heat loss within domestic buildings.

The entire thermal balance system is the foundation of the thermal model. In next sections, the mathematical schemas of both PB and SB thermal models will be explained in details.

\section{Physics-Based (PB) Estimation Model}

A mathematical formulation of the problem will take a general form to display the internal connections between amounts of energy input and output. Thus, due to the first law of thermodynamics [17], the mathematical schema of 
the PB thermal model which describes the total energy exchange within the thermal zone is given by the following:

$\frac{d Q}{d t}=Q_{\text {in }}(t)-Q_{\text {out }}(T, t)$,

where the function $Q_{\text {in }}(t)$, which is dependent on time only, encompasses all information related to heat generation, and the function $Q_{\text {out }}(T, t)$, which is dependent on both time and temperature, denotes all information regarding to heat loss.

However, it is more convenient to measure the temperature rather than the quantities of heat within a thermal compartment. Considering the experimental compartment as a heat reservoir with uniform temperature, hence, on the basis of the physical relations between temperature and energy, $\Delta Q=m_{\text {air }} \times C_{\text {air }} \times \Delta T$, where $m_{\text {air }}$ and $C_{\text {air }}$ are the total inner mass of the air in $\mathrm{kg}$ and the heat capacity of air in $\mathrm{J} / \mathrm{kg} \cdot{ }^{\circ} \mathrm{C}$, respectively, Eq. 1 can be represented as follows:

$\frac{d T_{\text {in }}}{d t}=\frac{1}{m_{\text {air }} \times C_{\text {air }}} \times\left(Q_{\text {in }}(t)-Q_{\text {out }}(T, t)\right)$,

where $T_{\text {in }}$ denotes the internal temperature. Equation 2 indicates the relationships between factors which affect the temperature variation within a building in overview. It is obvious that the rate of the temperature varying is positive proportion to the differences between the heat generation and the heat loss, which conforms to the reality. Furthermore, the mass of the air in the thermal zone, $m_{\text {air }}$, can be determined by the mass formulas. Specifically, for a cubic compartment without a roof (adopted in the tests), the air mass $m_{\text {air }}=V_{\text {air }} \times \rho=l \times w \times h \times \rho$, where $l, w$ and $h$ in $m$ represent the dimension values of the building, and $\rho$ is the density of air in $\mathrm{kg} / \mathrm{m}^{3}$.

Assuming that the thermal zone is air conditioned by a conventional HS with effective equivalent power rate of $\dot{P}(t)$ in $k W$, the amounts of the input energy in a unit time $\Delta t$ can be calculated from the work-energy theorem as follows:

$Q_{\text {in }}(t)=\dot{P} \times \Delta t \times \eta$,

where $\eta$ is the thermal conversion efficiency of the given HS.

Equally important, the heat loss happens in every second. Regarding the thermal compartment that possesses the finest air impermeability and the most heat loss that comes from the heat conduction through the accessible surfaces in this study, the quantity of the output energy function, $Q_{\text {out }}(T, t)$, which is dependent on time and temperature, can be expressed as follows:

$Q_{\text {out }}(T, t)=R \times\left(T_{\text {in }}(t)-T_{\text {out }}(t)\right)$,

where variables $T_{\text {in }}$ and $T_{\text {out }}$ express internal temperature and external temperature, respectively. Additionally, $R$ in $\mathrm{hr} \cdot{ }^{\circ} \mathrm{C} / \mathrm{J}$ is treated as the equivalent thermal resistance of the building, which is a core parameter that refers to the ability of impeding temperature rising during the heating process. Besides, the conductive heat loss can be thought in terms of heating flowing from a hot region to a cold region through any surface components, such as walls or glass windows. Hence, the total equivalent resistance $R$ can be derived as a combination of each individual resistance in a series that is given in Eq. 5:

$R=\sum_{i=1}^{n} R_{i}$

where $n$ represents the number of considered conductive components in the model. In addition, according to the thermal conductive formula, $R_{i}=\gamma_{i} \times a_{i} / l_{i}$, where $\gamma$ in $\mathrm{J} / \mathrm{hr}$. $\mathrm{m} \cdot{ }^{\circ} \mathrm{C}$ is the heat conductive coefficient which is an inherent attribute of the conductive component and the value is only dependent on the type of conductive material. Parameters $a$ in $\mathrm{m}^{2}$ and $l$ in $m$ represent the conductive areas and the thickness of the corresponded conductive material, respectively. Afterwards, assume that the estimation process is divided into a set of discrete time periods with a time interval $t_{s}$. Thus, according to Eqs. $2-4$, the $(k+1)^{\text {th }}$ internal temperature can be modelled as follows:

$$
\begin{aligned}
T_{\mathrm{in}}^{(k+1)} & =\Delta T_{\mathrm{in}}+T_{\mathrm{in}}^{(k)} \\
& =\frac{t_{s}}{m_{\mathrm{air}} \times C_{\mathrm{air}}} \times\left(Q_{\mathrm{in}}^{(k)}-Q_{\mathrm{out}}^{(k)}\right)+T_{\mathrm{in}}^{(k)} \\
& =\lambda \times\left(\dot{P} \times \eta-R \times\left(T_{\mathrm{in}}^{(k)}-T_{\mathrm{out}}^{(k)}\right)\right) \times t_{s}+T_{\mathrm{in}}^{(k)},
\end{aligned}
$$

where $\lambda=\frac{1}{m_{\text {air }} \times C_{\text {air }}}$. Meanwhile, $T_{\text {in }}{ }^{(k)}$ and $T_{\text {out }}{ }^{(k)}$ represent the $k^{\text {th }}$ transient internal and external temperature, respectively.

For a conventional PB model, the related physical parameters are often obtained directly by checking the handbook and the field measurement. Moreover, the values of the relevant parameters which will be used in the model are listed in Table 1. In accordance with the proposed mathematical schema and the determined coefficients, the PB model is capable of simulating the thermal dynamics within a building and the corresponded internal temperature variation can be estimated.

In spite of that, the PB thermal model is not perfect and there are still areas for improvement. Specifically, a drawback of relying on this type of physical attribute-based thermal model is that it requires a great number of parameters, describing the properties of the building, to be determined in advance. Although some of the parameters can be achieved directly by field measurement, some of these parameters are difficult to obtain, such as the parameters related to the 
Table 1 Values of the adopted house parameters in PB estimation model

\begin{tabular}{lll}
\hline ID & Meaning & Value \\
\hline$a_{1}$ & Wall areas $\left(\mathrm{m}^{2}\right)$ & 52.31 \\
$l_{1}$ & Wall thickness $(\mathrm{m})$ & 0.44 \\
$\gamma_{1}$ & Wall conductive coefficient $\left(\mathrm{W} / \mathrm{m} \cdot{ }^{\circ} \mathrm{C}\right)$ & $0.02-0.04$ \\
$a_{2}$ & Window areas $\left(\mathrm{m}^{2}\right)$ & 9.53 \\
$l_{2}$ & Window thickness $(\mathrm{m})$ & $0.10-0.25$ \\
$\gamma_{2}$ & Window conductive coefficient $\left(\mathrm{W} / \mathrm{m} \cdot{ }^{\circ} \mathrm{C}\right)$ & 0.20 \\
$P$ & Heater rated power $(\mathrm{kW})$ & $1.95-2.05$ \\
$\eta$ & Conversion rate & $0.10-0.25$ \\
$\rho$ & Air density $\left(\mathrm{kg} / \mathrm{m}^{3}\right)$ & $1.19-1.24$ \\
$C$ & Air heat capacity $\left(\mathrm{J} / \mathrm{kg} \cdot{ }^{\circ} \mathrm{C}\right)$ & 1005.4 \\
\hline
\end{tabular}

characteristics of the construction materials since the composite materials are widely adopted in building construction recently. As a consequence, the proposed PB model with imprecise parameters may lead to considerable prediction errors in some cases. Therefore, in order to improve the performance of the model and reduce the temperature estimation errors, an innovative SB model is proposed in next section.

\section{Semi-blind (SB) Estimation Model}

As presented in "Physics-Based (PB) Estimation Model", the core parameter determination becomes an essential optimisation problem in model establishing. In this section, the proposed SB model will focus on parameter optimisation and improving the temperature estimation performance. Both self-adaptive algorithms (SAAs) and grey prediction technique (GPT) are used in integrated parameter estimation (IPE) process.

\section{Problem Formulation}

For a certain building, due to the stable physical characteristics, such as the spacial structure and the construction materials, the thermal insulation property of the building should be relatively stable and will seldom change over time. Hence, the equivalent thermal resistances which are affected by these permanent physical attributes can be considered as a unity. Moreover, the quantity of the heat generation is mostly caused by heating devices. An electrical heater with fixed power rate is used in our tests. Thus, the heat generation in a unit time can be regarded as stable as well. However, $\lambda=\frac{1}{m_{\text {air }} \times C_{\text {air }}}$ in Eq. 6 is independently and identically distributed. $C_{\text {air }}$ and $m_{\text {air }}$ cannot be determined easily and precisely in practice, since the mass of the air and the heat capacity vary with the environment. Therefore, a group of integrated parameters, $\left[\phi_{1}, \phi_{2}\right]$ are proposed to illustrate the relationships between various factors, where $\phi_{1}=\lambda \times R$ and $\phi_{2}=\lambda \times \dot{P} \times \eta$. By using the integrated parameters, the SB estimation model can be given as follows:

$$
T_{\text {in }}^{(k+1)}=\left[\phi_{2}-\phi_{1} \times\left(T_{\text {in }}^{(k)}-T_{\text {out }}^{(k)}\right)\right] \times t_{s}+T_{\text {in }}^{(k)} .
$$

Hence, the effects of $m_{\text {air }}$ and $C_{\text {air }}$ are hidden in $\phi_{1}$ and $\phi_{2}$, which are explicit and can be obtained using statistical techniques. The advantages of this assumption are as follows: (i) The complicated factors affecting the heat generation and heat loss are not necessary to be investigated individually. They are considered as a unity and can be estimated based on data-driven approaches. (ii) The model becomes more adaptable for various type of buildings, since the integrated parameter estimation (IPE) is only dependent on the collected temperature data of the objective building.

\section{Parameter Estimation}

The integrated parameter estimation (IPE) is the most significant task in establishing a thermal model, as the accuracy of the parameter estimation directly determines the forecasting performance of the model. The IPE of the model includes two phases: cooling estimation phase (CEP) and heating estimation phase (HEP). In CEP, the HS is turned off so that $Q_{\text {in }}=0$. The rate of the internal temperature decreasing is only affected by the quantity of lost energy which is closely related to $\phi_{1}$. Nonetheless, in HEP, the heat preservation and radiation happen simultaneously. Thus, the quantity of both energy generation (related to $\phi_{2}$ ) and loss (related to $\phi_{1}$ ) has decisive impacts on the internal temperature variation. Therefore, the thermal-temperature model represented in Eq. 7 can be explain in two phases as below.

Phase 1: Temperature estimation in CEP

$$
T_{\mathrm{in}, \mathrm{c}}^{(k+1)}=T_{\mathrm{in}, \mathrm{c}}^{(k)}-\phi_{1}^{(t)} \times\left(T_{\mathrm{in}, \mathrm{c}}^{(k)}-T_{\mathrm{out}, \mathrm{c}}^{(k)}\right) \times t_{s}
$$

Phase 2: Temperature estimation in HEP

$$
T_{\mathrm{in}, \mathrm{h}}^{(k+1)}=T_{\mathrm{in}, \mathrm{h}}^{(k)}+\left[\phi_{2}^{(t)}-\phi_{1}^{(t)} \times\left(T_{\mathrm{in}, \mathrm{h}}^{(k)}-T_{\mathrm{out}, \mathrm{h}}^{(k)}\right)\right] \times t_{s}
$$

To obtain the parameters, some approaches such as exhaustive searching [18] can be used for reference. However, the exhaustive searching method iterating through every possible solutions needs much time; it is not appropriate in this case. Therefore, a numerical parameter estimation 
method is adopted in this study. By using the recursion formulas of temperature estimation in Eqs. 8 and 9, the formulas for the general term of the model can be derived in Eqs. 10 and 11 (the detailed derivation can be seen in Appendix A).

$T_{\mathrm{in}, \mathrm{c}}^{\left(k_{1}\right)}=-\phi_{1}^{(t)} \times \int_{t_{s}}^{k_{1} \times t_{s}}\left(\hat{T}_{\mathrm{in}, \mathrm{c}}-\hat{T}_{\mathrm{out}, \mathrm{c}}\right) d t+T_{\mathrm{in}, \mathrm{c}}^{(1)}$

$T_{\mathrm{in}, \mathrm{h}}^{\left(k_{2}\right)}=\left(k_{2}-1\right) \times \phi_{2}^{(t)} \times t_{s}-\phi_{1}^{(t)} \int_{t_{s}}^{k_{2} \times t_{s}}\left[\hat{T}_{\mathrm{in}, \mathrm{h}}-\hat{T}_{\mathrm{out}, \mathrm{h}}\right] d t+T_{\mathrm{in}, \mathrm{h}}^{(1)}$,

where $T_{\text {in,c }}^{\left(k_{1}\right)}$ represents the $k_{1}^{\text {th }}$ predicted temperature in CEP. Similarly, $T_{\mathrm{in}, \mathrm{h}}^{\left(k_{2}\right)}$ denotes the $k_{2}^{\text {th }}$ predicted temperature in HEP. In addition, $\phi_{1,2}^{(t)}$ represents the corresponded parameters at temperature point $t$. The temperature interval is assumed as $0.1^{\circ} \mathrm{C}$. Moreover, the variations of the internal and external temperature in CEP and HEP are defined as $\left[\hat{T}_{\mathrm{in}, \mathrm{c}}, \hat{T}_{\mathrm{out}, \mathrm{c}}\right]$ and $\left[\hat{T}_{\mathrm{in}, \mathrm{h}}, \hat{T}_{\mathrm{out}, \mathrm{h}}\right]$, respectively.

According to the literature, the undetermined functions $\hat{T}_{\text {in,c }}, \hat{T}_{\text {out }, \mathrm{c}}, \hat{T}_{\mathrm{in}, \mathrm{h}}$ and $\hat{T}_{\text {out, h }}$ can be achieved by using the least square estimation (LSE) technique [19]. The method of LSE is a standard approach in regression analysis to the approximate solution of over determined system [20]. It is one of the fitting algorithms and is widely used in both linear and non-linear systems [21]. For example, since the collected data is a set of discrete points, assuming that $\hat{T}_{\mathrm{in,c}}$ is a linear polynomial function varying with time as follows:

$\hat{T}_{\mathrm{in,c}}(t)=\sum_{i=0}^{n} \alpha_{i} t^{i}$

where the $\alpha_{i}$ 's are the parameters (also called weights) parameterizing the space of linear functions mapping from $\mathcal{X}$ to $\mathcal{Y}$ and let $A^{T}=\left[\alpha_{1}, \alpha_{2}, \ldots, \alpha_{i}\right]$. The polynomial function is not the unique option in this case. Despite that, in line with the characteristics of polynomial function, it can be an appropriate choice in this study. Thus, vector $A$ can be resolved by minimising the objective function as shown below:

$\min E_{S} \mid A=\frac{1}{2} \sum_{k=1}^{n}\left\|T_{\mathrm{in}, \mathrm{c}}^{(k)}-\hat{T}_{\mathrm{in}, \mathrm{c}}^{(k)}\right\|^{2}$,

where $T_{\text {in,c }}^{(k)}$ and $\hat{T}_{\text {in,c }}^{(k)}$ are the vectors of actual and estimated temperature values. According to the methodology of LSE, parameter vector $A$ can be obtained by taking partial derivative of $E_{S}$ with respect to each element in $A$ (the derivation for obtaining polynomial functions in general form can be found in Appendix B). Similarly, all the functions can be achieved in turn by adopting LSE periodically.

Hence, the integrated parameters $\phi_{1}^{(t)}$ and $\phi_{2}^{(t)}$ at a specifical temperature $t$ (e.g. $t=21.5^{\circ} \mathrm{C}$ ) can be calculated as follows:

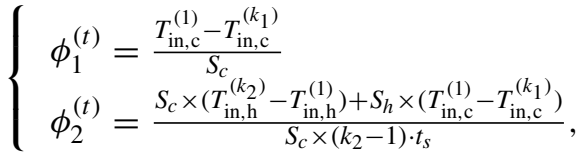

where $S_{c}=\int_{t_{s}}^{k_{1} \times t_{s}}\left(\hat{T}_{\mathrm{in}, \mathrm{c}}-\hat{T}_{\text {out }, \mathrm{c}}\right) d t$ and $S_{h}=\int_{t_{s}}^{k_{2} \times t_{s}}\left(\hat{T}_{\mathrm{in}, \mathrm{h}}-\right.$ $\left.\hat{T}_{\text {out }, \mathrm{h}}\right) d t . S_{c}$ and $S_{h}$ can be approximately regarded as the areas of $\left[\hat{T}_{\mathrm{in}, \mathrm{c}}, \hat{T}_{\mathrm{out}, \mathrm{c}}\right]$ and $\left[\hat{T}_{\mathrm{in}, \mathrm{h}}, \hat{T}_{\mathrm{out}, \mathrm{h}}\right]$, respectively.

\section{Parameter Analysis}

\section{SAAs for Parameter Selection}

A number of integrated parameters at temperature $t$ can be acquired by using multiple sets of data. However, some of parameter samples are not accurate due to the unexpected experimental mistakes, and it will cause considerable estimation errors by accounting them into the model. Hence, self-adaptive algorithms (SAAs) for parameter selection are proposed to investigate the optimal parameters among all the samples. The selection strategy refers to the customers' behaviours and being executed persistently with the using time increasing.

First, we define a vector to describe the parameter samples at temperature point $t$ as $\Phi_{t}=\left[\phi_{t}^{(1)}, \phi_{t}^{(2)}, \ldots, \phi_{t}^{(i-1)}\right]$ and variable $S$ as the objective optimal parameter. Assuming that $\phi_{t}^{(i)}$ is the next input sample and variable $m$ is a threshold which denotes the capacity of samples. Second, define $\sigma^{(i)}$ as the standard deviation (SD) of $i^{\text {th }}$ sample, $\sigma_{\max }^{(n)}$ as the maximum SD among all samples, and $w^{(i)}$ as the weighing coefficient (WC) of $i^{\text {th }}$ sample. The weighing coefficient reflects the risk of being an unexpected sample. The higher value of WC denotes the lower risk and greater impact in parameter estimation. Further, the initial optimal parameter $S$, is given as a mean value of the input vector with $m$ samples as follows:

$S=\frac{1}{m} \sum_{j=1}^{m} \phi_{t}^{(j)}$

In SAAs, the mean square deviation method [22, 23] and weighted average method $[24,25]$ are used. Therefore, the SAAs can be illustrated as the pseudo-code below. 


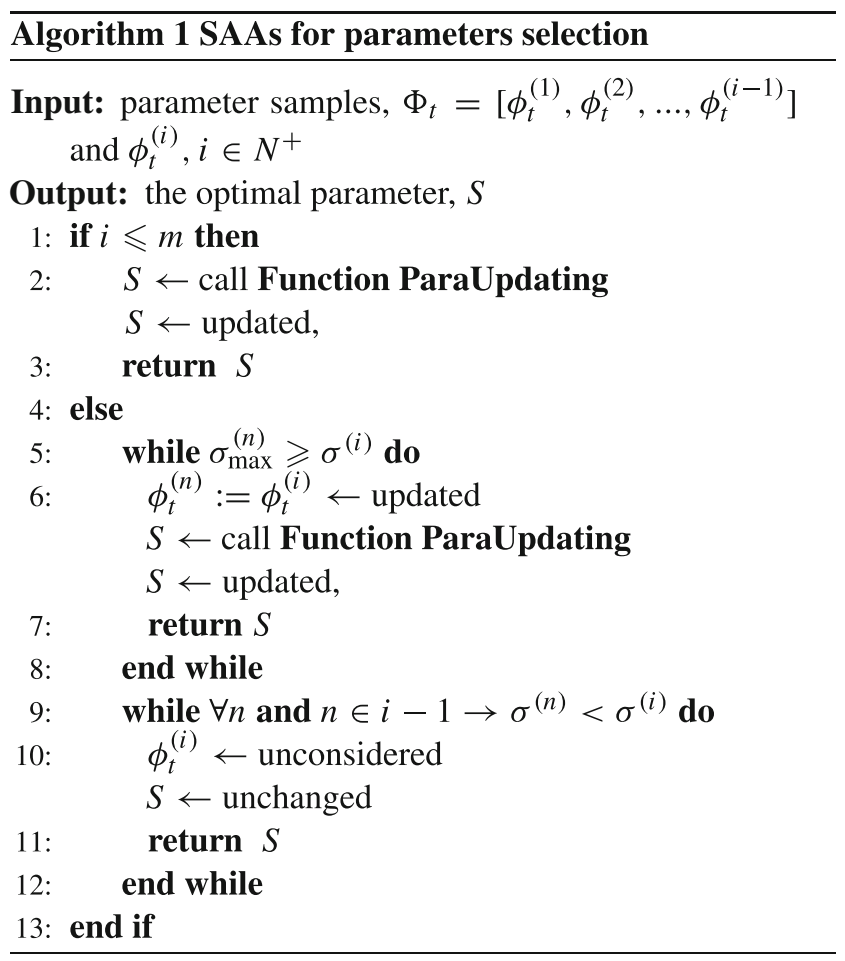

Here, we use the notation " $A:=b$ " to denote an operation in which we set the value of a variable $a$ to be equal to the value of $b$. The proposed parameter self-adaptive strategy enables the model to renew along with time and the parameters tend to stabilisation if the experiencing time is long enough. Additionally, SAAs are able to reduce the system errors caused by unexpected samples, thus improving the accuracy of temperature estimation.

However, the parameter estimation in SB model is dependent on temperature range of collected data, which means the relevant parameters $\phi_{t}$ cannot be obtained, when $t \nsubseteq$ $\left[T_{a}, T_{b}\right]$, where $\left[T_{a}, T_{b}\right]$ is the temperature range of the data. Next, we will present how to forecast the imponderable parameters by using grey prediction techniques (GPT).

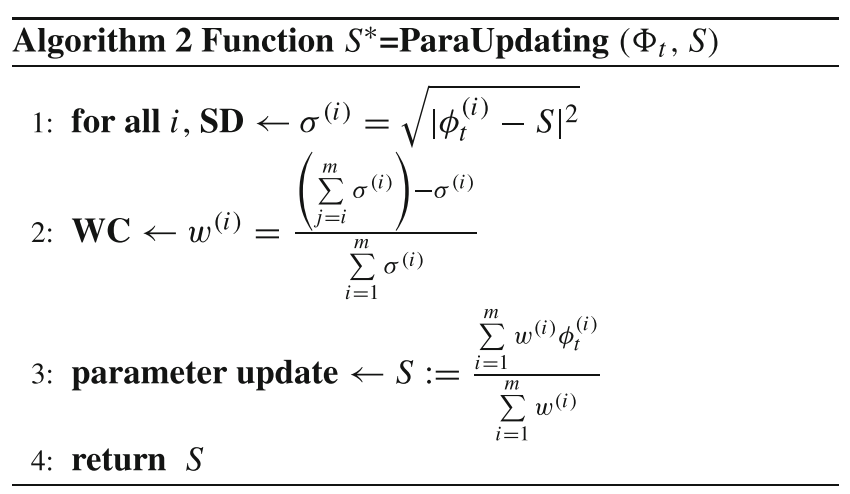

\section{Imponderable Parameter Forecasting}

The imponderable parameter which means the parameter that cannot be calculated by using the data-driven methods, since the restriction of data range. Nonetheless, it is can be hypothesised that there are some internal relations between these imponderable parameters and the obtained integrated parameters. Thus, the imponderable parameter is able to be evaluated on the basis of the achieved parameters by using GPT.

The GPT was first proposed to deal with the data in grey systems. It is able to analyse the system that includes insufficient information and unapparent relationship [26, 27]. Hence, to predict some unknown information based on limited information, GPT is often used. Moreover, it is accounted for that irregular discrete sequences of numbers are some display of potential regular sequences. Transforming the forms of the sequences can make the properties of stochastic and randomness get weaker thereby turning irregular sequences to regular ones [28, 29]. Since there are limited parameter samples and the relations between each sample are undefined in our case, it is quite appropriate to employ the GPT to forecast the imponderable parameters.

The GPT model is established by using generalised series. The primitive sequence data is given in $X_{0}$ :

$X_{0}=\left\{x_{0}^{(1)}, x_{0}^{(2)}, \ldots, x_{0}^{(n)}\right\}$,

where $x_{0}^{(k)} \geqslant 0, k=1,2, \ldots, n, n \subseteq N^{+}$.

Afterwards, the first accumulated generating data $X_{1}$ can be obtained according to accumulating generation operator (AGO):

$X_{1}=\left\{x_{1}^{(1)}, x_{1}^{(2)}, \ldots, x_{1}^{(n)}\right\}$,

where $x_{1}^{(k)}=\sum_{i=1}^{k} x_{0}^{(i)}, k=1,2, \ldots, n, n \subseteq N^{+}$.

Define $Z_{1}$ as the background factors determined by $X_{1}$, $Z_{1}$ can be presented as follows:

$Z_{1}=\left\{z_{1}^{(1)}, z_{1}^{(2)}, \ldots, z_{1}^{(n)}\right\}$,

where $z_{1}^{(k)}=\frac{1}{2}\left[x_{1}^{(k)}+x_{1}^{(k-1)}\right], k=2,3, \ldots, n, n \subseteq N^{+}$.

In $X_{0}$, if any $x_{0}^{(k)}<0$ in primitive sequence samples, all the candidates in the sequence have to be improved until $\forall x_{0}^{(k)} \geqslant 0$.

Furthermore, after first accumulated generating operation towards $X_{0}$, the new sequence $X_{1}$ is provided with the quasi exponential property and $X_{1}$ should be satisfied with the first-order ordinary differential equation which is shown in Eq. 16:

$\frac{d X_{1}}{d t}+a X_{1}=u$, 
where $a$ is regarded as a development coefficient that describes the increasing speed of numbers in $x^{(0)}$ and $u$ is an endogenous control coefficient in system. Moreover, the parameters $U=[a, u]^{T}$ can be determined in Eq. 17 .

$U=\left(B^{T} B\right)^{-1} B^{T} Y_{n}$,

where $Y_{n}$ is a $(n-1) \times 1$ matrix and $B$ is a $(n-1) \times 2$ matrix. $Y_{n}$ and $B$ can be presented as follows:

$Y_{n}=\left[\begin{array}{c}x_{0}^{(2)} \\ x_{0}^{(3)} \\ \vdots \\ x_{0}^{(n)}\end{array}\right]$ and $B=\left[\begin{array}{cc}-z_{1}^{(2)} & 1 \\ -z_{1}^{(3)} & 1 \\ \vdots & \vdots \\ -z_{1}^{(n)} & 1\end{array}\right]$.

Then, resolve the Eq. 16 by using the obtained $a$ and $u$, and the prediction formula of $\hat{X}_{1}$ can be denoted as follows:

$\hat{x}_{1}^{(k+1)}=\left(x_{1}^{(1)}-\frac{u}{a}\right) e^{-a k}+\frac{u}{a}$.

In Eq. 18 , when $k=1,2, \ldots, n-1, \hat{x}^{(1)}(k+1)$ is a fitted value. When $k \geqslant n, \hat{x}^{(1)}(k+1)$ is a predicted value of $X_{1}$. Afterwards, the predicted formula of $\hat{X}_{0}$ can be achieved through inverse accumulating generation operator (IAGO) in Eq. 19.

$$
\begin{aligned}
\hat{x}_{0}^{(k+1)} & =\hat{x}_{1}^{(k+1)}-\hat{x}_{1}^{(k)} \\
& =\left(1-e^{a}\right)\left[x_{1}^{(1)}-\frac{u}{a}\right] e^{-a k}
\end{aligned}
$$

Therefore, we are able to forecast the imponderable parameters by employing GPT model periodically based on the achieved parameter samples.

By using the SB model and the achieved parameters, the internal temperature variations are able to be estimated. In the next section, a number of experiments are carried out to evaluate the feasibility of the proposed model.

\section{Experimental Study}

To specifically evaluate the implementation of the proposed thermal models, a number of experiments and simulations are carried out by using an illustrative medium size office building. This is a free reference-building provided by Department of Electrical and Electronic Engineering, Xi' an Jiaotong-Liverpool University. In data-collecting phase, we recorded 2 months of minutely data (from Nov. 15, 2016 to Jan. 15, 2017) with various environment conditions for the internal and external temperature of the given building. The capacity of the data set is over $500 \mathrm{~h}$ in total. In experiments, a brand new electric radiator with a rated power of $2 \mathrm{~kW}$ is used as the heat generation source and several electronic thermometers with minimum precision of $0.1{ }^{\circ} \mathrm{C}$ are used for temperature recording.

At the outset of experiment, the relevant building information has to be identified. Afterwards, the numerical results are presented and the estimation performances of the proposed PB and SB model are compared with the actual measured data.

\section{Setup}

In this study, we investigate the application of optimisation methods (e.g. SAAs and GPT) for parameter identification of a SB building thermal model. In order to evaluate the performance of the proposed approach for a real building, the values of requisite relevant building parameters have to be acquired, to ensure that the models operate correctly. Additionally, the benchmark needs to be set up for performance comparison as well in this study.

\section{PB Estimation Model Identification}

For a PB estimation model, the following building parameters are considered:

- Dimensions: structure, length $l$, width $w$ and height $h$;

- Accessible conduction components : types, areas $a_{i}$, thickness $l_{i}$ and heat conduction coefficient $\gamma_{i}$;

- Inner air characteristics: air heat capacity $C_{\text {air }}$ and air density $\rho$;

- HS specification: rated power $P$ and thermal conversion efficiency $\eta$.

It is not difficult to obtain the physical parameters (e.g. dimensions) of the building by using traditional measurement methods. In addition, the parameters related to the conduction materials can be achieved by checking building handbook and references. Table 1 illustrates some core parameters that have been used in this study.

\section{SB Estimation Model Identification}

In contrast to PB model, we do not consider the complicated factors within building in SB model. The SB model is established by using historical temperature data which can be showed below.

- Multiple sets of internal temperature data, $T_{\text {in }}$, in both CEP and HEP with time in Celsius;

- Corresponding external temperature data, $T_{\text {out }}$, with time in Celsius;

Meanwhile, the historical temperature data of $T_{\text {in }}$ and $T_{\text {out }}$ are recorded by the electronic thermometers with sensitive 
Table 2 Estimated parameters' values in SB estimation model at the temperature point $t=21^{\circ} \mathrm{C}$

\begin{tabular}{lllllllll}
\hline Data index & 1 & 2 & 3 & 4 & 5 & 6 & 7 & 8 \\
\hline$\phi_{1}^{\times 10^{-3}}$ & 4.89 & 4.61 & 5.44 & 4.98 & 4.17 & 4.77 & 4.25 & 4.11 \\
$\phi_{2}^{\times 10^{-2}}$ & 8.72 & 9.38 & 8.21 & 8.69 & 7.70 & 7.21 & 8.19 & 8.44 \\
Data index & 9 & 10 & 11 & 12 & 13 & 14 & 15 & 16 \\
$\phi_{1}^{\times 10^{-3}}$ & 5.07 & 5.41 & 3.99 & 4.54 & 4.41 & 4.87 & 4.98 & 4.62 \\
$\phi_{2}^{\times 10^{-2}}$ & 9.74 & 8.52 & 8.41 & 8.54 & 8.63 & 9.45 & 8.12 & 7.93 \\
Data index & 17 & 18 & 19 & 20 & 21 & 22 & 23 & 24 \\
$\phi_{1}^{\times 10^{-3}}$ & 4.55 & 4.63 & 4.75 & 4.82 & 4.37 & 4.62 & 4.21 & 5.11 \\
$\phi_{2}^{\times 10^{-2}}$ & 8.74 & 8.23 & 8.91 & 8.04 & 8.32 & 8.98 & 7.65 & 8.67 \\
\hline
\end{tabular}

sensors. On the basis of data, SB thermal model can be activated.

\section{Benchmark Setup}

The root mean square deviation (RMSD) is taken as the benchmark for temperature estimation result evaluation in this study. In statistics, RMSD is a frequently used measure of the differences between values predicted by a model or an estimator and the values actually observed. Hence, it is an appropriate benchmark in this case. The RMSD is always none-negative and it can be defined by the formula as follows:

$\operatorname{RMSD}=\sqrt{\frac{\sum_{i=1}^{n}\left(\hat{y}_{i}-y_{i}\right)^{2}}{n}}$,

where $y_{i}$ is the observed value and $\hat{y}_{i}$ is the predicted value. The RMSD value closer to zero means a better performance of the proposed estimator.

\section{Numerical Results}

First of all, to evaluate the feasibility of SAAs in SB estimation model, the threshold $m$ is assumed as $m=5$ in the evaluation. In addition, 24 sets of estimated parameters (obtained according to Eq. 14) at a specific temperature point (e.g. $t=21^{\circ} \mathrm{C}$ ) which are demonstrated in Table 2, are randomly selected for the simulation.

As a result, Fig. 3 shows the performance of the estimated parameters after self-adapting based on SAAs. According to the results, we can see that both $\phi_{1}$ and $\phi_{2}$ tend to be stable after self-adaptive process. Specifically, in Fig. 3a, the estimated parameter $\phi_{1}$ with SAAs ranges from $4.75 \times 10^{-3}$ to $4.98 \times 10^{-3}$ and gradually stabilised at $4.90 \times 10^{-3}$. Similarly, in Fig. $3 b$, the estimated parameter $\phi_{2}$ with SAAs ranges from $8.54 \times 10^{-2}$ to $8.75 \times 10^{-2}$ and becomes stable at $8.59 \times 10^{-2}$. Furthermore, it is obvious that some unexpected parameter values (e.g. $\phi_{1}=5.41 \times 10^{-3}$, index $=10$ and $\phi_{2}=9.45 \times 10^{-2}$, index $=14$ ) are self-filtered by SAAs, thus reducing the system errors apparently. The trends of parameter variation in results verify the analysis in previous sections and prove that the SB estimation model tends to be more stable and accurate with SAAs with the using time increasing.

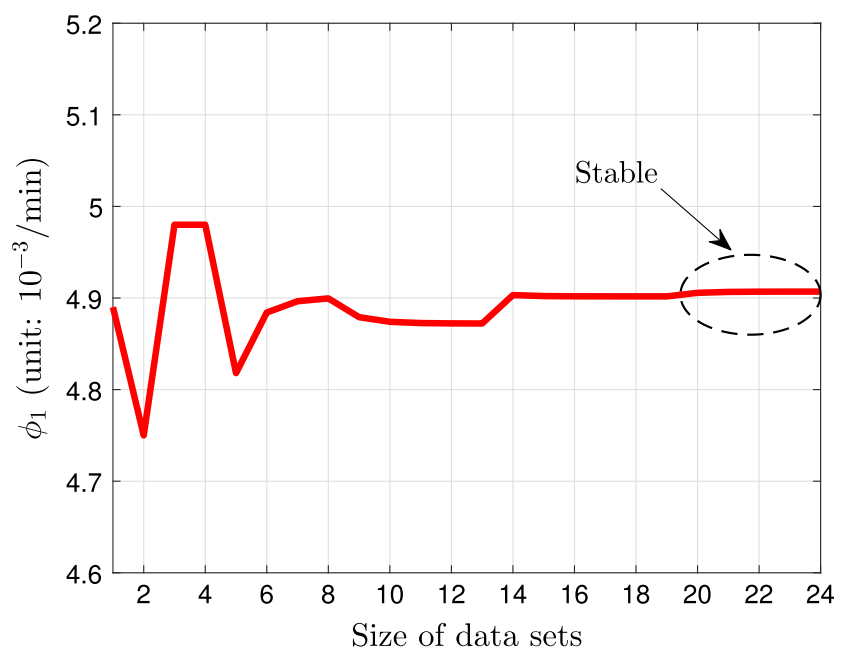

(a) SAAs performance of $\phi_{1}$

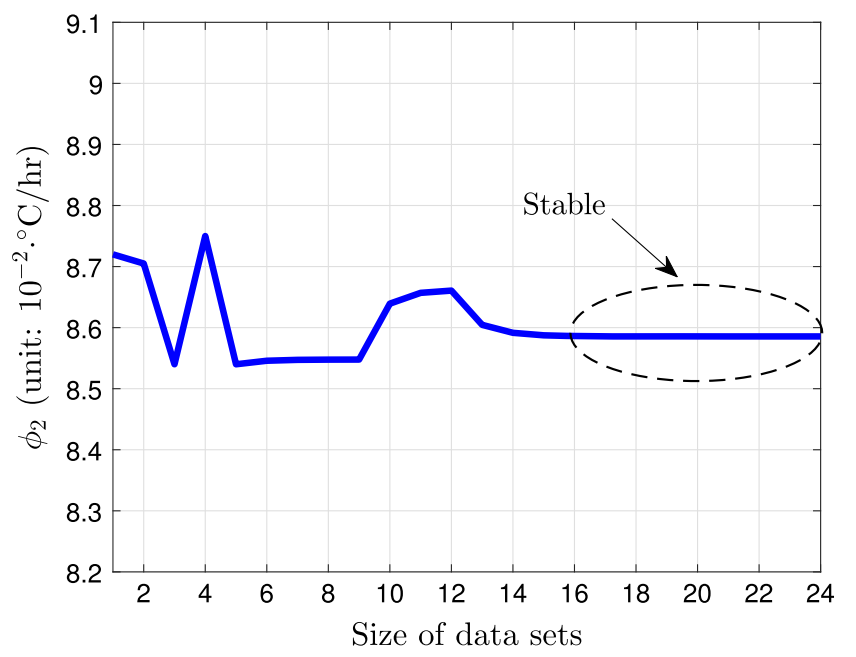

(b) SAAs performance of $\phi_{2}$

Fig. 3 Parameter self-adaptive performances evaluation based on SAAs 


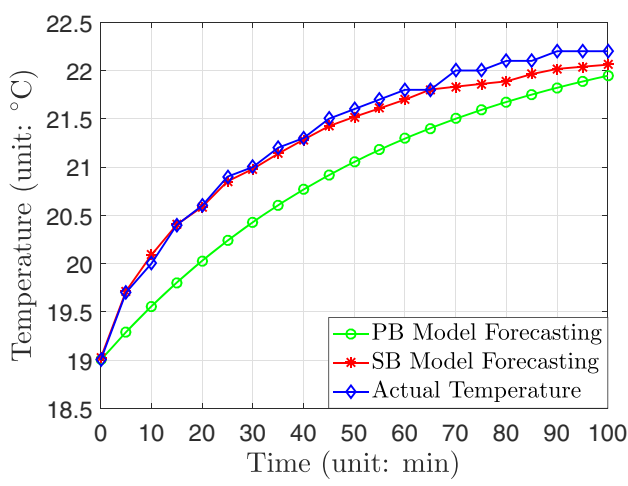

(a) Mean external temperature: $T_{\text {out }}=5.9^{\circ} \mathrm{C}$

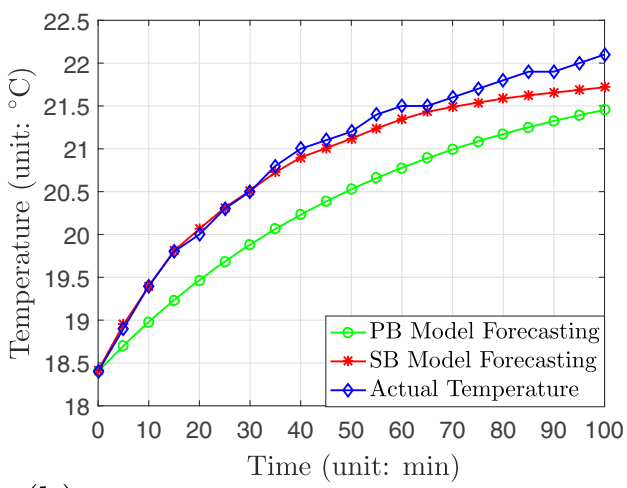

(b) Mean external temperature: $T_{\text {out }}=6.7^{\circ} \mathrm{C}$

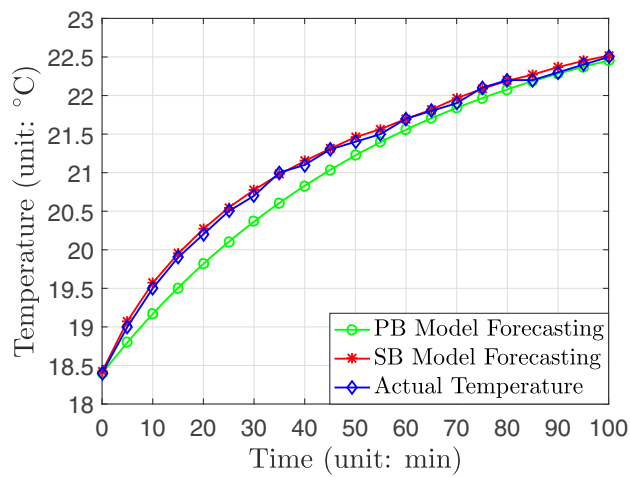

(c) Mean external temperature: $T_{\text {out }}=9.8^{\circ} \mathrm{C}$

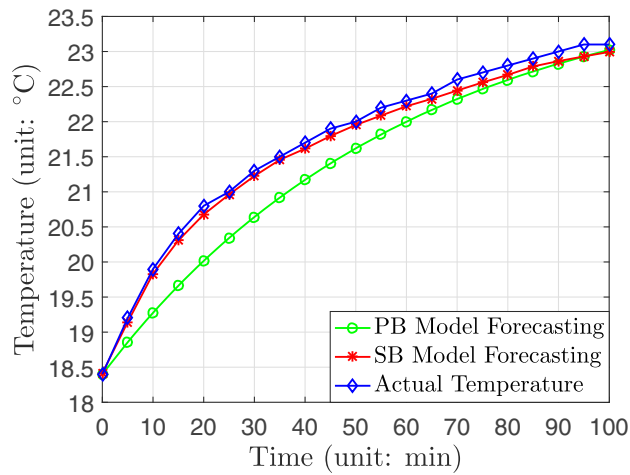

(d) Mean external temperature: $T_{\text {out }}=10.1^{\circ} \mathrm{C}$

Fig. 4 The estimating results comparison under different external temperature conditions in HEP

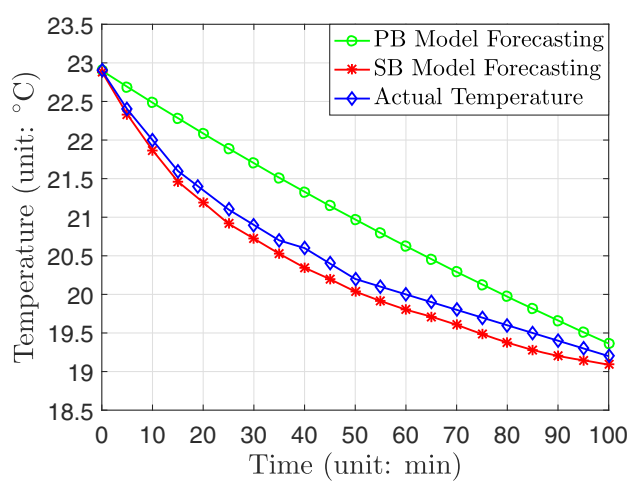

(a) Mean external temperature: $T_{\text {out }}=7.1^{\circ} \mathrm{C}$

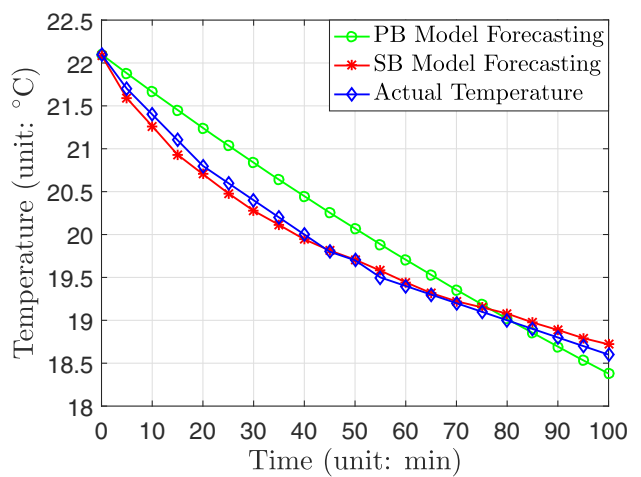

(b) Mean external temperature: $T_{\text {out }}=8.7^{\circ} \mathrm{C}$

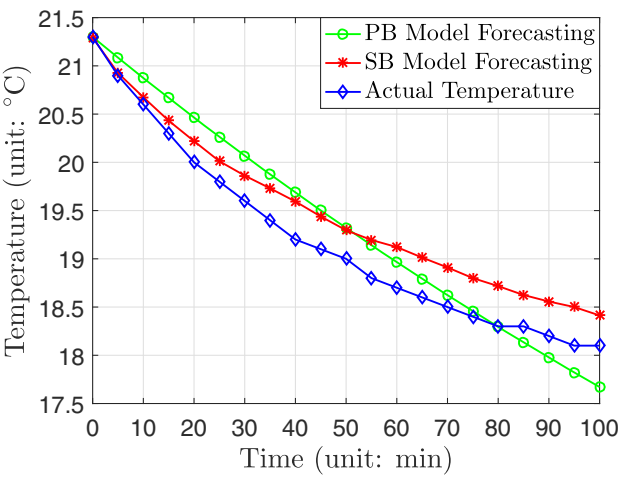

(c) Mean external temperature: $T_{\text {out }}=10.0^{\circ} \mathrm{C}$

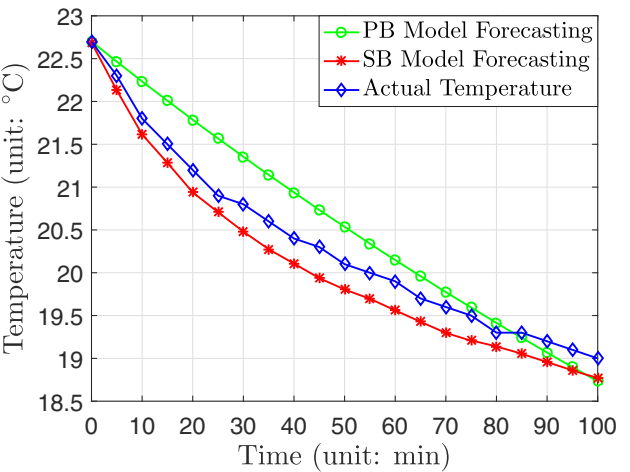

(d) Mean external temperature: $T_{\text {out }}=11.4^{\circ} \mathrm{C}$

Fig. 5 The estimating results comparison under different external temperature conditions in CEP 
Additionally, the internal temperature estimation results by using PB model and SB model under various external temperature conditions in HEP and CEP are presented in Figs. 4 and 5, respectively. The external conditions are randomly selected from the database and the mean external temperature ranges from 5.9 to $11.4{ }^{\circ} \mathrm{C}$ in this study. Based on the obtained results, it is apparent that the estimating curves by SB estimation model are well fitted to the measured ones in both HEP and CEP. On the other hand, the PB estimation model performs well in HEP, but there are considerable errors occurring in CEP. These errors may be caused by using the inaccurate physical parameters in the model.

Moreover, the estimating performance of SB model is better than PB model according to the numerical results in overview. The RMSD of SB model and PB model are 0.18 and 0.43 , respectively. Since SB model is developed on the basis of PB model, in accordance with the obtained results, it can be summarised that great improvement has been achieved comparing with the original one.

\section{Conclusion}

The aim of this work is to develop an optimal thermal model for better understanding of thermal dynamics with the goal of using this to estimate temperature variation in a few hours ahead within building. Based on the achieved results, we can conclude that the proposed $\mathrm{PB}$ and SB models are able to appropriately estimate the internal temperature values and the performance of SB model is better than that in PB model. The RMSD of SB model and PB model are 0.18 and 0.43 , respectively, which means great improvement has been achieved in this work. Nonetheless, we only considered an individual compartment or neighboring compartments with similar environment conditions as research object in this work. Hence, one improvement can be accounting more complicated building structures into the research that may lead to more challenges in next stage. The proposed models have wide potential applications, such as better controlling of HVAC system and reducing energy cost with high quality of using experience.

Acknowledgements This work is supported by the University of Liverpool and Xi' an Jiaotong-Liverpool University.

\section{Compliance with Ethical Standards}

Ethical Approval This article does not contain any studies with human participants performed by any of the authors.

Conflict of Interest The authors declare that they have no conflict of interest.
Informed Consent Informed consent was obtained from all individual participants included in the study.

Open Access This article is distributed under the terms of the Creative Commons Attribution 4.0 International License (http:// creativecommons.org/licenses/by/4.0/), which permits unrestricted use, distribution, and reproduction in any medium, provided you give appropriate credit to the original author(s) and the source, provide a link to the Creative Commons license, and indicate if changes were made.

Appendix A: The Derivation of $T_{\mathrm{in}, \mathrm{c}}^{(k)}$ and $T_{\mathrm{in}, \mathrm{h}}^{(k)}$

$$
\begin{aligned}
& T_{\mathrm{in}, \mathrm{c}}^{(k)}=-\phi_{1}^{(t)} \times\left(T_{\mathrm{in}, \mathrm{c}}^{(k-1)}-T_{\mathrm{out}, \mathrm{c}}^{(k-1)}\right) \times t_{s}+T_{\mathrm{in}, \mathrm{c}}^{(k-1)} \\
& =-\phi_{1}^{(t)} \times\left[\left(T_{\mathrm{in}, \mathrm{c}}^{(k-1)}+T_{\mathrm{in}, \mathrm{c}}^{(k-2)}\right)-\left(T_{\mathrm{out}, \mathrm{c}}^{(k-1)}+T_{\mathrm{out}, \mathrm{c}}^{(k-2)}\right)\right] \\
& \times t_{s}+T_{\text {in, }}^{(k-2)} \\
& =-\phi_{1}^{(t)} \times\left[\sum_{n=1}^{k-1}\left(T_{\text {in, }}^{(n)}-T_{\text {out }, \mathrm{c}}^{(n)}\right) \times t_{s}\right]+T_{\text {in }, \mathrm{c}}^{(1)} \\
& =-\phi_{1}^{(t)} \times \int_{t_{s}}^{k \times t_{s}}\left(\hat{T}_{\mathrm{in}, \mathrm{c}}-\hat{T}_{\text {out }, \mathrm{c}}\right) d t+T_{\mathrm{in}, \mathrm{c}}^{(1)} \\
& T_{\mathrm{in}, \mathrm{h}}^{(k)}=\phi_{2}^{(t)} t_{s}-\phi_{1}^{(t)} \times\left(T_{\mathrm{in}, \mathrm{h}}^{(k-1)}-T_{\mathrm{out}, \mathrm{h}}^{(k-1)}\right) \times t_{s}+T_{\mathrm{in}, \mathrm{h}}^{(k-1)} \\
& =2 \phi_{2}^{(t)} t_{s}-\phi_{1}^{(t)} \times\left[\left(T_{\mathrm{in}, \mathrm{h}}^{(k-1)}+T_{\mathrm{in}, \mathrm{h}}^{(k-2)}\right)-\left(T_{\mathrm{out}, \mathrm{h}}^{(k-1)}+T_{\mathrm{out}, \mathrm{h}}^{(k-2)}\right)\right] \\
& \times t_{s}+T_{\mathrm{in}, \mathrm{h}}^{(k-2)}
\end{aligned}
$$

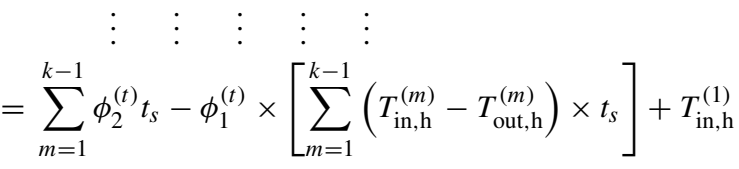

$$
\begin{aligned}
& =(k-1) \phi_{2}^{(t)} t_{s}-\phi_{1}^{(t)} \int_{t_{s}}^{k \times t_{s}}\left(\hat{T}_{\mathrm{in}, \mathrm{h}}-\hat{T}_{\mathrm{out}, \mathrm{h}}\right) d t+T_{\mathrm{in}, \mathrm{h}}^{(1)}
\end{aligned}
$$

\section{Appendix B: The Derivative of Solving Polynomial Functions in General Term}

$$
\begin{aligned}
\frac{\partial E_{s}}{\partial \alpha_{l}} & =\sum_{k=1}^{n}\left[T_{\mathrm{in}, \mathrm{c}}^{(k)}-\left(\alpha_{0}+\alpha_{1} t^{(k)}+\ldots+\alpha_{i} t^{(k) i}\right)\right] \times\left(-t^{(k) l}\right) \\
& =-\sum_{k=1}^{n}\left[T_{\mathrm{in}, \mathrm{c}}^{(k)} t^{(k) l}-\left(\alpha_{0} t^{(k) l}+\alpha_{1} t^{(k) l+1} \ldots+\alpha_{i} t^{(k) l+i}\right)\right]
\end{aligned}
$$


where $l=0,1, \ldots, i, i \in N^{+}$. Let $\frac{\partial E_{s}}{\partial \alpha_{l}}=0$, then we have the following:

$\sum_{k=1}^{n} T_{\mathrm{in}, \mathrm{c}}^{(k)} t^{(k) l}=\sum_{k=1}^{n}\left(\alpha_{0} t^{(k) l}+\alpha_{1} t^{(k) l+1}+\ldots+\alpha_{i} t^{(k) l+i}\right)$

Afterwards, transform Eq. 24 into matrix form:

$\sum_{k=1}^{n}\left(\left[\begin{array}{ccc}t^{(k) 0} & \cdots & t^{(k) i} \\ \vdots & \ddots & \vdots \\ t^{(k) i} & \cdots & t^{(k) 2 i}\end{array}\right] A^{T}\right)=\sum_{k=1}^{n}\left[\begin{array}{c}T_{i c}^{(k)} t^{(k) 0} \\ \vdots \\ T_{i c}^{(k)} t^{(k) i}\end{array}\right]$

As a result, the parameter vector $A$ can be obtained by multiplying the inverse matrix on both sides of Eq. 25. Thus, the vector $A$ is proposed as follows:

$A^{T}=\left[\begin{array}{ccc}\sum_{k=1}^{n} t^{(k) 0} & \cdots & \sum_{k=1}^{n} t^{(k) i} \\ \vdots & \ddots & \vdots \\ \sum_{k=1}^{n} t^{(k) i} & \cdots & \sum_{k=1}^{n} t^{(k) 2 i}\end{array}\right]^{-1}\left[\begin{array}{c}\sum_{k=1}^{n} T_{i c}^{(k)} t^{0(k)} \\ \vdots \\ \sum_{k=1}^{n} T_{i c}^{(k)} t^{i(k)}\end{array}\right]$

*all required variables $\left[T_{\mathrm{in}, \mathrm{c}}^{(k)}, t^{(k)}, n\right]$ in Eq. 26 can be found in database.

\section{References}

1. Han J, Choi Cs, Park Wk, Lee I, Kim Sh. Smart home energy management system including renewable energy based on ZigBee and PLC. IEEE Trans Consum Electron. 2014;60(2):198-202.

2. Prez-Lombard L, Ortiz J, Pout C. A review on buildings energy consumption information. Energy Build. 2008;40(3):394-8.

3. Wen Y, Burke W. Real-time dynamic house thermal model identification for predicting HVAC energy consumption. In: 2013 IEEE Green Technologies Conference. Denver, Colorado; 2013. p. 367-72.

4. De Felice M, Yao X. Short-term load forecasting with neural network ensembles: a comparative study [application notes]. IEEE Comput Intell Mag. 2011;6(3):47-56.

5. Oldewurtel F, Jones CN, Parisio A, Morari M. Stochastic model predictive control for building climate control. IEEE Trans Control Syst Technol. 2014;22(3):1198-205.

6. Yang Z, Li X, Bowers CP, Schnier T, Tang K, Yao X. An efficient evolutionary approach to parameter identification in a building thermal model. IEEE Trans Syst, Man, Cybern, Part C Appl Rev. 2012;42(6):957-69.

7. Park H, Ruellan M, Bouvet A, Monmasson E, Bennacer R. Thermal parameter identification of simplified building model with electric appliance. In: 2011 11th International Conference on Electrical Power Quality and Utilisation (EPQU). Lisbon, Portugal; 2011. p. 1-6.

8. Mallikarjun S, Gautam AR, Muniyasamy K, Maharaja M, Subathra B, Srinivasan S. Lasso based building thermal model for heating, ventilation and air-conditioning control. In: IEEE International Conference on Electrical, Computer and Communication Technologies (ICECCT). Coimbatore, India; 2015. p. 1-6. 2015.
9. Xu L, Ding S, Xu X, Zhang N. Self-adaptive extreme learning machine optimized by rough set theory and affinity propagation clustering. Cogn Comput. 2016;8(4):720-8.

10. Khamassi I, Sayed-Mouchaweh M, Hammami M, Ghédira K. Self-adaptive windowing approach for handling complex concept drift. Cogn Comput. 2015;7(6):772-90.

11. Boaro M, Fuselli D, Angelis FD, Liu D, Wei Q, Piazza F. Adaptive dynamic programming algorithm for renewable energy scheduling and battery management. Cogn Comput. 2013;5(2):264-77.

12. Gao Y, Fan R, Zhang Q-L, Roux JJ. Building dynamic thermal simulation of low-order multi-dimensional heat transfer. J Central South Univ. 2014;21(1):293-302.

13. Ma Y, Borrelli F, Hencey B, Coffey B, Bengea S, Haves P. Model predictive control for the operation of building cooling systems. IEEE Trans Control Syst Technol. 2012;20(3):796-803.

14. Skruch P. A general fractional-order thermal model for buildings and its properties. Heidelberg: Springer International Publishing; 2013, p. 213-220.

15. Qin M, Yang J. Evaluation of different thermal models in EnergyPlus for calculating moisture effects on building energy consumption in different climate conditions. Build Simul. 2016;9(1): $15-25$.

16. Xiong G, Kundu A, Kundu A, Fisher TS. Thermal modeling of supercapacitors. Cham: Springer International Publishing; 2015, p. 115-141.

17. Vaghefi SA, Jafari MA, Zhu J, Brouwer J, Lu Y. A hybrid physics-based and data driven approach to optimal control of building cooling/heating systems. IEEE Trans Autom Sci Eng. 2016;13(2):600-10.

18. Hashemi Y, Banihashemi AH. On characterization and efficient exhaustive search of elementary trapping sets of variable-regular LDPC codes. IEEE Commun Lett. 2015;19(3):323-26.

19. Abebe AT, Kang CG. Iterative order recursive least square estimation for exploiting frame-wise sparsity in compressive sensingbased MTC. IEEE Commun Lett. 2016;20(5):1018-21.

20. Yutthagowith $P$, Pattanadech N. Improved least-square prony analysis technique for parameter evaluation of lightning impulsevoltage and current. IEEE Trans Power Deliv. 2016;31(1):271-7.

21. Guilin T, Yunming Q. Improved least square method apply in ship performance analysis. In: Proceedings 2010 3rd International Conference on Advanced Computer Theory and Engineering (ICACTE). Chengdu, China; 2010. vol. 5, p. 594-596.

22. Jeong JJ, Kim SH, Koo G, Kim SW. Mean-square deviation analysis of multiband-structured subband adaptive filter algorithm. IEEE Trans Signal Process. 2016;64(4):985-94.

23. Liu Y. Mean square error of survey estimates. Netherlands, Dordrecht: Springer; 2014. p. 3892-3893.

24. Azis NA, Jeong YS, Choi HJ, Iraqi Y. Weighted averaging fusion for multi-view skeletal data and its application in action recognition. IET Comput Vis. 2016;10(2):134-42.

25. Bonfietti A, Lombardi M. The weighted average constraint. Berlin, Heidelberg: Springer Berlin Heidelberg; 2012. p. 191-206.

26. Wu L, Liu S, Yang Y. A gray model with a time varying weighted generating operator. IEEE Trans Syst, Man, Cybern Syst. 2016;46(3):427-33.

27. Gao X, Huang T, Wang Z, Xiao M. Exploiting a modified gray model in back propagation neural networks for enhanced forecasting. Cogn Comput. 2014;6.

28. Zeng B, Li C, Chen G, Long X. Equivalency and unbiasedness of grey prediction models. J Syst Eng Electron. 2015;26(1):1108.

29. Xie N, Liu S. Interval grey number sequence prediction by using non-homogenous exponential discrete grey forecasting model. J Syst Eng Electron. 2015;26(1):96-102. 